trypsine, soit normale, soit mutée; on a pu les purifier et en comparer les propriétés. Toutes deux se montrent capables d'inhiber l'élastase, mais seul le mutant résiste à l'oxydation expérimentale par la $\mathrm{N}$-chlorosuccinimide. On a donc pu fabriquer, par mutagenèse dirigée, une protéine mutée plus stable que la protéine naturelle. Est-il possible d'en prévoir une utilisation thérapeutique?

L'emploi de l' $\alpha_{1}$ antitrypsine est envisagé dans les déséquilibres élastase-antiélastase : avant tout les déficits en antitrypsine, mais aussi d'autres types d'emphysème en cours d'identification. Les quantités requises sont élevées. Peut-être précisément du fait de son oxydabilité $l^{\prime} \alpha_{1}$ antitrypsine a une demi-vie de 4 à 5 jours, très inférieure à celle des principales protéines du plasma. Pour traiter un déficient on estime qu'il faudrait lui fournir environ $4 \mathrm{~g}$ d'antitrypsine par semaine (le plasma en contient vingt fois moins que d'albumine). Une stabilisation de la protéine aurait donc un intérêt pratique considérable.

Nature a publié en janvier 1985 un article des chercheurs de Transgène (Strasbourg). Utilisant une technique analogue, ils ont eux aussi préparé une antitrypsine à valine. De plus, mettant à profit le cas pathologique évoqué plus haut, ils ont remplacé méthionine par arginine et obtenu une activité inhibitrice de la thrombine. Ils espèrent ainsi aboutir à un succès sans précédent : l'exploitation pour la thérapeutique d'une mutation pathologique.

Ces résultats ouvrent des perspectives extrêmement prometteuses, et il est probable que cette "évolution provoquée" sera source de thérapeutiques nombreuses et encore imprévisibles aujourd'hui. J.-C.-D.

Rosenberg S, Barr PJ, Najarian RC, Hallewel RA. Synthesis in yeast of a functional oxidationresistant mutant of human $\alpha_{1}$ antitrypsin. Nasure 1984; $312: 77-80$.

Owen MC, Brennan SO, Lewis JH, Carrell RW. Mutation of antitrypsin to antithrombin: $\alpha_{1}$ Antitrypsin Pittsburgh (358 met $\rightarrow$ arg), a fatal blecding disorder. New Engl 7 Med 1984; 309: 694-8.

Courtney M, Tessier L H, Benavente A, Crystal $\mathbf{R}$, Lecocq J P. Synthesis in $E$. coli of $\alpha_{1}$ antitrypsin variants of therapcutic potential for emphysema and thrombosis. Nature 1985; 313 : 149-51.

\title{
L'avènement des ribozymes
}

$\mathbf{Q}$ uelques anciens se souviennent encore d'une controverse qui fit rage au début du siècle dans les milieux scientifiques : les enzymes sont-elles des protéines, ou bien les protéines ne sont-elles que le support inerte de l'activité catalytique ? Vers 1930 la question était réglée et le monopole des protéines sur la catalyse en biologie fermement établi. Tout au plus pouvaient-elles accepter parfois un auxiliaire, qualifié de coenzyme ou de groupement prosthétique.

Récemment, un nouveau compétiteur est entré dans l'arène, l'acide ribonucléique. Pour le moment sa candidature reste encore modeste : parmi les trois types principaux d'ARN, comme substrat il ne s'adresse qu'à l'ARN ribosomique et à l'ARN de transfert, pas encore au messager.

Ce sont les investigations de Thomas Cech et de son équipe à Boulder (Colorado) qui ont ouvert cette voie. Travaillant sur le mécanisme d'épissage des introns contenus dans le précurseur de l'ARN ribosomique chez un protozoaire cilié, Tetrahymena thermophila, ils ont montré que cet ARN pouvait à lui seul exciser ces introns, en l'absence de tout catalyseur protéique. Une fois excisé, l'intron se cyclisait rapidement, prévenant ainsi toute possibilité de réinsertion du fragment détaché.

Maintenus en veilleuse pendant plus de deux ans, ces résultats sont aujourd'hui largement reconnus. Mais les biochimistes, gens pointilleux, renâclaient à accorder le terme de catalyse à l'action de l'ARN ribosomique : un catalyseur authentique, on l'apprend à l'école, doit se retrouver inchangé et prêt à agir à nouveau au sortir de la réaction. L'ARN ribosomique de Cech est raccourci par son action elle-même et a perdu tout pouvoir catalytique. Or depuis dix ans, Sidney Altman (Yale) se débattait pour faire accepter l'idée d'une activité enzymatique de l'ARN. Il travaillait sur une enzyme dénommée ribonucléase $P$, dont la spécificité est de scinder les précurseurs des ARN de transfert pour les amener à l'état mature et fonctionnel. La ribonucléase $P$ contient cinq fois plus d'ARN que de protéine, mais s'inactivait lorsqu'on séparait les deux composants. L'affirmation que l'ARN devait être la fraction active se heurtait à l'incrédulité polie des enzymologistes. La solution fut obtenue grâce à l'obstination de l'équipe et à un coup de chance. Comme il est classique en enzymologie on essaya d'augmenter l'activité des préparations en faisant varier les conditions expérimentales. On utilisa ainsi des concentrations variables de magnésium auxquelles on ajouta l'enzyme complète contenant à la fois ARN et protéine. En même temps, on mit en œuvre des " témoins négatifs" contenant seulement, soit l'ARN, soit la protéine, qu'on s'attendait à trouver inactifs. Or les tubes contenant l'ARN seul, mais en présence d'une concentration élevée de magnésium, s'avérèrent actifs. A partir de là, les preuves de l'activité enzymatique de l'ARN s'accumulèrent.

Nous l'avons dit, la ribonucléase P d'Altman et al. est une enzyme bactérienne. Mais on n'en restera certainement pas là. Dans chaque cellule on a dénombré quantité de petites molécules d'ARN qui attendent qu'on leur assigne une fonction. Il a d'ores et déjà été montré qu'un ARN intervient dans la biosynthèse de l'acide $\delta$ aminolévulinique chez des végétaux (où il représente le facteur limitant dans la synthèse de la chlorophylle, comme chez les animaux dans celle de l'hème). L'intervention de l'ARN en tant que catalyseur ajouterait une pierre à cet édifice qui donne à ce type de molécule un rôle éminent dans les premières manifestations de la vie. Elle corrobore en tous cas l'extraordinaire flexibilité de l'ARN, capable de se plier à toutes les tâches biologiques plus aisément que l'ADN et les protéines. Les ribozymes ont donc devant elles un avenir prometteur. J.-C. D.

Cech TR et al. Nature 1984; $301: 578-83 /$ Lewin R. Science $1984 ; 223: 266-7 /$ Altman S, et al. Science 1984; $223: 285-6 /$ Huang DD, et al. Science $1984 ; 225: 1482-4$. 